TITLE:

\title{
Possible spin singlet quadrumerization in $\mathrm{Pb}(2) \operatorname{Re}(2) \mathrm{O}(7-$ $\delta)$.
}

AUTHOR(S):

Michioka, C; Kataoka, Y; Ohta, H; Yoshimura, K

\section{CITATION:}

Michioka, C ... [et al]. Possible spin singlet quadrumerization in $\mathrm{Pb}(2) \operatorname{Re}(2) \mathrm{O}(7-\delta)$.. Journal of physics. Condensed matter 2011, 23(44): 445602.

\section{ISSUE DATE:}

2011-11-09

URL:

http://hdl.handle.net/2433/151719

\section{RIGHT:}

(c) IOP Publishing 2011; This is not the published version. Please cite only the published version.; この論文は出版社版でありません。引用の 際には出版社版をご確認ご利用ください。 


\title{
Possible spin singlet quadrumerization in $\mathrm{Pb}_{2} \mathrm{Re}_{2} \mathrm{O}_{7-\delta}$
}

\author{
C. Michioka, Y. Kataoka, H. Ohta, K. Yoshimura \\ Department of Chemistry, Graduate School of Science, Kyoto University, Kyoto \\ 606-8502, Japan \\ E-mail: michioka@kuchem.kyoto-u.ac.jp
}

\begin{abstract}
A single crystal of pyrochlore $\mathrm{Pb}_{2} \mathrm{Re}_{2} \mathrm{O}_{6.75}$ was prepared by hydrothermal synthesis, and its magnetic and transport properties were investigated. $\mathrm{Pb}_{2} \operatorname{Re}_{2} \mathrm{O}_{6.75}$ shows the metallic conduction with an anomalous second order structural phase transition at $T_{\mathrm{S}}=294 \mathrm{~K}$, in which the magnetization and the structure change similarly to that at $200 \mathrm{~K}$ of the superconductor $\mathrm{Cd}_{2} \mathrm{Re}_{2} \mathrm{O}_{7}$. A three-dimensional Ising-like order parameter probed by the atomic displacement suggests that an unconventional spin singlet state is possibly realized in the ground state of $\mathrm{Pb}_{2} \mathrm{Re}_{2} \mathrm{O}_{6.75}$, in which four spins make a quadrumer in order to remove the spin frustration of the pyrochlore lattice.
\end{abstract}

PACS numbers: 75.47.Lx, 75.40.Cx, 75.40.-s 


\section{Introduction}

Magnetic frustration sometimes causes exotic phase transitions with coupling with another interaction. The tetrahedron is the minimum unit to exhibit strong spin frustration effects in the three-dimensional system as well as the triangular cell in the two dimensional one in frustrated spin systems. Pyrochlore lattice is one of the most ideal models for investigating three-dimensional geometrical frustration. As shown in Fig. 1 (a), the lattice points form a three-dimensional network of tetrahedrons. The pyrochlore oxides show a variety of ground states: spin liquid or valence bond crystal for the localized spin systems and superconductivity or charge ordering for the itinerant electron systems. For example, $\mathrm{Cd}_{2} \mathrm{Re}_{2} \mathrm{O}_{7}$ exhibits superconductivity at $T_{\mathrm{C}}=1.1 \mathrm{~K}$ with two structural phase transitions at $T_{\mathrm{S} 1}=200 \mathrm{~K}$ and $T_{\mathrm{s} 2}=120 \mathrm{~K}$, in which the degenerate energy levels by geometrical frustration are separated by a perturbation energy from the lattice $[1,2] . \mathrm{Tl}_{2} \mathrm{Ru}_{2} \mathrm{O}_{7}$ shows a metal to insulator transition (MIT) by forming an unconventional spin singlet state [3, 4]. Another ruthenium pyrochlore oxide, $\mathrm{Hg}_{2} \mathrm{Ru}_{2} \mathrm{O}_{7}$, also shows a similar MIT [5]. Fujimoto proposed that MITs in the pyrochlore [6] and the spinel lattices $[7,8]$ occur as a result of an interplay between geometrical frustration and electron correlations [9]. From a viewpoint of frustrated metals, experimental studies of physical properties in the novel itinerant pyrochlore oxides are very important. However, unfortunately many pyrochlore oxides are insulating, and only a few pyrochlore oxides are itinerant. Among such pyrochlore oxides, $\mathrm{Pb}_{2} \mathrm{Re}_{2} \mathrm{O}_{7-\delta}$ shows a metallic resistivity as well as the superconductor $\mathrm{Cd}_{2} \mathrm{Re}_{2} \mathrm{O}_{7}$, and details of the physical properties are not clarified up to now.

In this paper, we report the magnetic and transport properties of the single crystal $\mathrm{Pb}_{2} \mathrm{Re}_{2} \mathrm{O}_{7-\delta}$ which was grown large enough for measurements of physical properties for the first time, and then unconventional characteristics of the observed second order structural phase transition are discussed from a viewpoint of geometrical frustration.

(a)

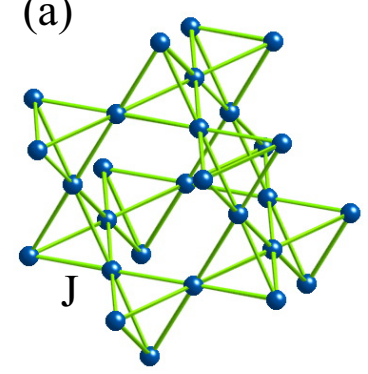

(b)

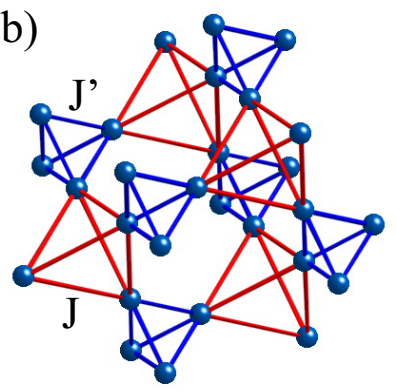

(c)

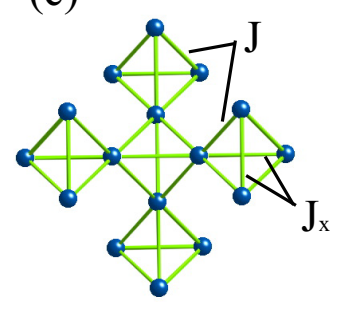

Figure 1. (a) Schematic drawing of the pyrochlore lattice, corresponding to the high temperature phase of $\mathrm{Pb}_{2} \mathrm{Re}_{2} \mathrm{O}_{6.75}$. (b) Low temperature phase of $\mathrm{Pb}_{2} \mathrm{Re}_{2} \mathrm{O}_{6.75}$. (c) Artificial model from Ref.[17], in which the ground state is spin singlet quadrumer in the condition of $J_{\times} / J \sim 1$. 


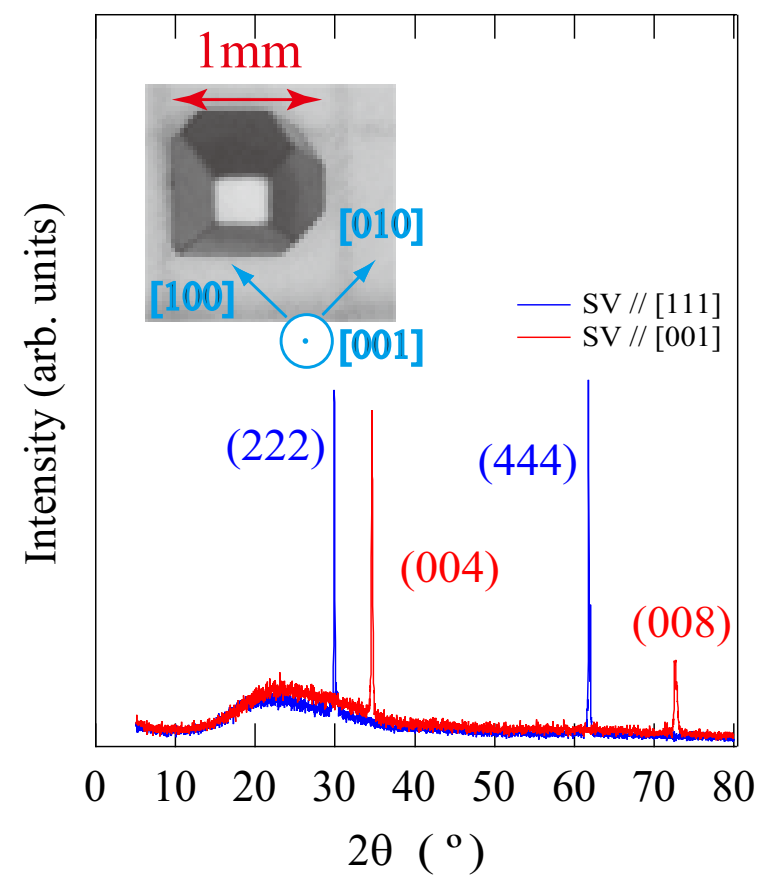

Figure 2. X-ray diffraction patterns of the single crystal $\mathrm{Pb}_{2} \mathrm{Re}_{2} \mathrm{O}_{6.75}$ with the scattering vectors $(S V)$ parallel to [100] and [111] axes at room temperature. Inset shows a photograph of the single crystal with crystal axes.

\section{Experiments and Sample Characterization}

The polycrystalline sample of $\mathrm{Pb}_{2} \mathrm{Re}_{2} \mathrm{O}_{7-\delta}$ was synthesized from a mixture of $\mathrm{Re}(99.99$ $\%), \mathrm{PbO}\left(99.99 \%\right.$ ) and previously prepared $\mathrm{ReO}_{3}$. The pellet of the pressed mixture was sintered at $600{ }^{\circ} \mathrm{C}$. Single crystals were grown by hydrothermal synthesis from the polycrystalline sample. Polycrystalline $\mathrm{Pb}_{2} \mathrm{Re}_{2} \mathrm{O}_{7-\delta}$ and $\mathrm{NaOH} a q$ were sealed in a silver tube and heated up to $600{ }^{\circ} \mathrm{C}$ with a maximum pressure of $1000 \mathrm{~kg} / \mathrm{cm}^{2}$ in an autoclave. The metallic-gray-colored single crystal was obtained with a size of $1 \times 1 \times 1 \mathrm{~mm}^{3}$.

A part of the single crystal was powdered for powder X-ray diffraction (XRD), which showed it to be a single phase of a cubic pyrochlore structure at room temperature. The lattice constant was found to be $a=10.424 \AA$. In common pyrochlore oxides $A_{2} B_{2} \mathrm{O}_{1-\delta}$, presence of oxygen defects generally depends on the $A$ site element. In the case of $A=$ $\mathrm{Pb}$, there is a certain amount of oxygen deficiency while the compounds with $A=\mathrm{Cd}$ can be obtained only with a stoichiometric ratio. By assuming the Vegard's law [10], the chemical formula of the single crystal was determined as $\mathrm{Pb}_{2} \mathrm{Re}_{2} \mathrm{O}_{6.75}$. In the pyrochlore oxides, there are two oxygen sites. Six oxygens are located in the $\mathrm{BO}_{6}$ octahedron and one near the $A$ cation. The oxygen deficiency occurs only at the latter site. These defects cause carrier doping effects on $5 d$-bands of Re because orbitals of Re hybridize mainly with those of oxygens in $\mathrm{BO}_{6}$ octahedrons. Therefore the band structure is not changed significantly, while the density of states is expected to be changed by such defects. Figure 2 shows the XRD patterns of the single crystal $\mathrm{Pb}_{2} \mathrm{Re}_{2} \mathrm{O}_{6.75}$ with the 
scattering vector parallel to [100] or [111] axis. In both conditions, only corresponding diffractions can be obtained without other diffractions, suggesting the single domain crystal. As shown in the inset of Fig. 2, the single crystal has edges corresponding to the crystallographic axes. The magnetization was measured with an applied field $H$ $=1 \mathrm{~T}$ using a superconducting quantum inference device magnetometer. The specific heat was measured by a relaxation method. The electric resistivity was measured by a conventional four probe method.

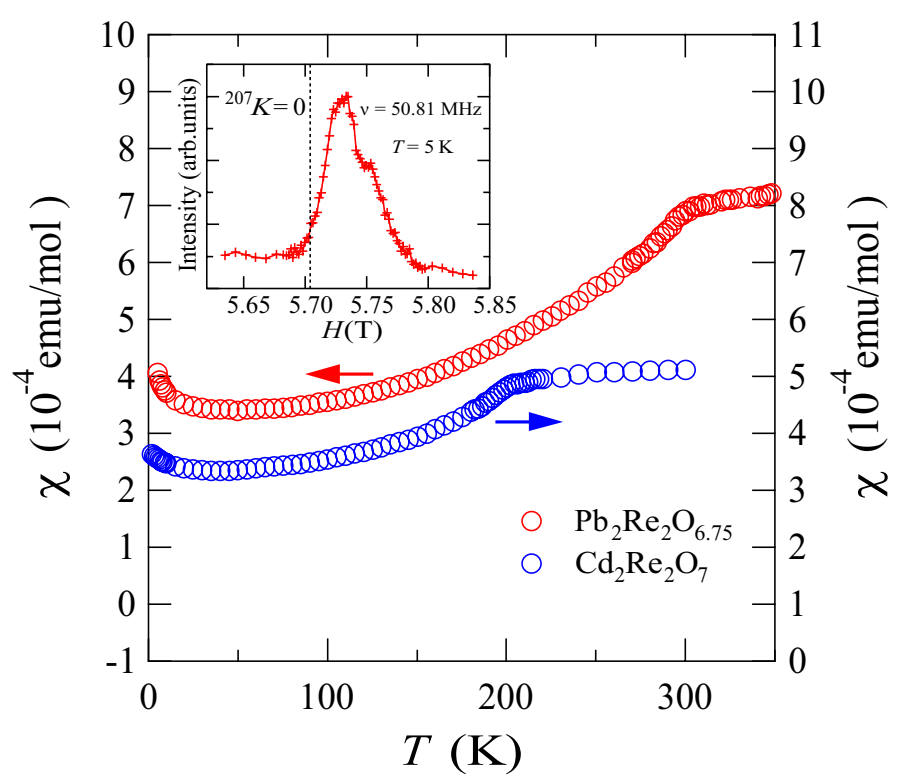

Figure 3. Temperature dependence of the magnetic susceptibilities $\chi$ of $\mathrm{Pb}_{2} \operatorname{Re}_{2} \mathrm{O}_{6.75}$ and $\mathrm{Cd}_{2} \mathrm{Re}_{2} \mathrm{O}_{7}$. Inset shows the field-swept ${ }^{207} \mathrm{~Pb} \mathrm{NMR}$ spectrum with a constant frequency $\nu=50.81 \mathrm{MHz}$ measured at $5 \mathrm{~K}$. No evidence for the magnetic ordering was observed down to $5 \mathrm{~K}$.

\section{Results and discussion}

Figure 3 shows the temperature dependence of the magnetic susceptibility $\chi$ in $\mathrm{Pb}_{2} \mathrm{Re}_{2} \mathrm{O}_{6.75}$ and that in $\mathrm{Cd}_{2} \mathrm{Re}_{2} \mathrm{O}_{7}$ for comparison. The data was measured with an external magnetic field $H$ parallel to [111]. There are not any anisotropic behaviors in $\chi$. In the case of $\mathrm{Pb}_{2} \mathrm{Re}_{2} \mathrm{O}_{6.75}, \chi$ shows an anomaly at about $300 \mathrm{~K}$ and then monotonically decreases with decreasing temperature below $300 \mathrm{~K}$. The small enhancement of $\chi$ below $10 \mathrm{~K}$ may be due to some impurities or defects. Except for the difference in the temperature at which $\chi$ shows a rapid drop, the temperature dependences of $\chi$ in $\mathrm{Pb}_{2} \mathrm{Re}_{2} \mathrm{O}_{6.75}$ and $\mathrm{Cd}_{2} \mathrm{Re}_{2} \mathrm{O}_{7}$ are very similar with each other. In the heat capacity measurement shown later, a second order phase transition was observed at $T_{\mathrm{s}}=294 \mathrm{~K}$ in $\mathrm{Pb}_{2} \mathrm{Re}_{2} \mathrm{O}_{6.75}$. The rapid decrease of $\chi$ is, therefore, due to a spin-singlet formation or the decrease of a density of states at the Fermi surface which is attributed to this transition. The inset of Fig. 3 shows the field-swept ${ }^{207} \mathrm{~Pb}$ NMR spectrum at $\nu=50.81$ 
$\mathrm{MHz}$ measured at $5 \mathrm{~K}$ in $\mathrm{Pb}_{2} \mathrm{Re}_{2} \mathrm{O}_{6.75}$. No broadening was not observed down to $5 \mathrm{~K}$, suggesting the absence of magnetic ordering.

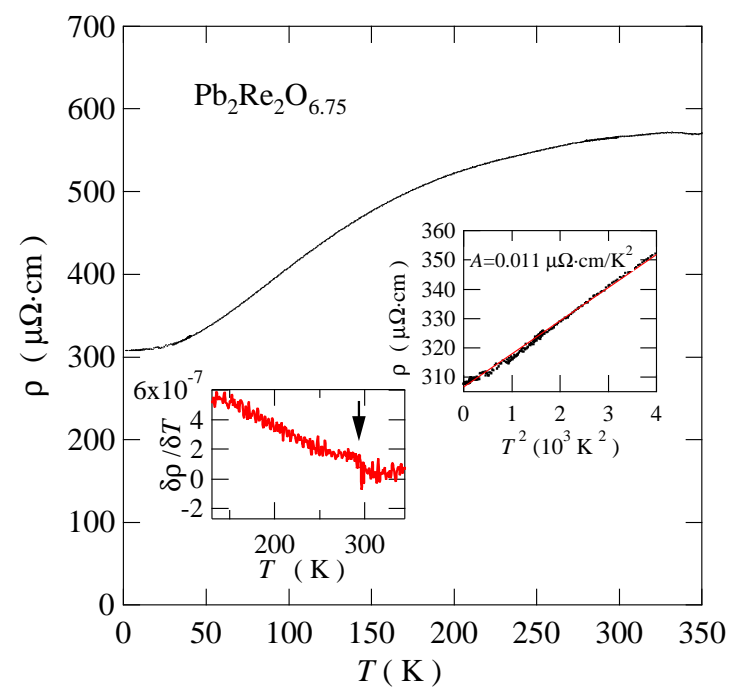

Figure 4. Temperature dependence of the electric resistivity of $\mathrm{Pb}_{2} \mathrm{Re}_{2} \mathrm{O}_{6.75}$. Left and right insets show the temperature dependence of $\delta \rho / \delta T$ and the plot of $\rho$ versus $T^{2}$, respectively.

The temperature dependence of the electric resistivity $\rho$ is shown in Fig. 4. With decreasing temperature, the resistivity gradually decreases. The temperature differential of resistivity as a function of temperature is shown in the left inset. The arrow indicates $T_{\mathrm{s}}$, at which the resistivity also shows the slight anomaly similar to the magnetic susceptibility and the heat capacity. In preliminary investigations, we found that some powder samples which contain small impurity phase obviously show recognizable anomalies at $T_{\mathrm{s}}$. To perform detailed studies, we need to make a larger single crystal and measure anisotropies of the resistivity. The residual resistance ratio (RRR) is found to be $\sim 2$. The small RRR is not due to poor sample quality but due to the intrinsic defects of oxygen sites. Superconductivity does not appear down to 0.8 $\mathrm{K}$ probably due to the oxygen defects in contrast to the stoichiometric $\mathrm{Cd}_{2} \mathrm{Re}_{2} \mathrm{O}_{7}$ with the superconducting transition temperature of about $1 \mathrm{~K}[1,2]$. The right inset shows the plot of $\rho$ versus $T^{2}$ below about $60 \mathrm{~K}$. In the wide temperature range, $\rho$ shows Fermi liquid-like behavior, i.e., $\rho$ varies in proportion to $T^{2}$. The solid line is a fit to the expression $\rho=\rho_{0}+A T^{2}$. The constant $A$ is $0.011 \mu \Omega \mathrm{cm} / \mathrm{K}^{2}$.

Figure 5 shows the temperature dependence of the heat capacity of $\mathrm{Pb}_{2} \mathrm{Re}_{2} \mathrm{O}_{6.75}$. As indicated by the arrow, clear second order phase transition is observed at $294 \mathrm{~K}$. The total change of entropy $\delta S$ accompanied by the phase transition is $\delta S=2.4 \mathrm{~J} / \mathrm{K}$ mol Re, which is comparable to $\delta S=3.5 \mathrm{~J} / \mathrm{K}$ mol Re for $\mathrm{Cd}_{2} \mathrm{Re}_{2} \mathrm{O}_{7}$ [11]. The lattice constant of $\mathrm{Pb}_{2} \mathrm{Re}_{2} \mathrm{O}_{6.75}$ at $T_{\mathrm{s}}$ is $2 \%$ larger than that of $\mathrm{Cd}_{2} \mathrm{Re}_{2} \mathrm{O}_{7}$ at $T_{\mathrm{s} 1}$. Compared with $\mathrm{Cd}_{2} \mathrm{Re}_{2} \mathrm{O}_{7}$, 


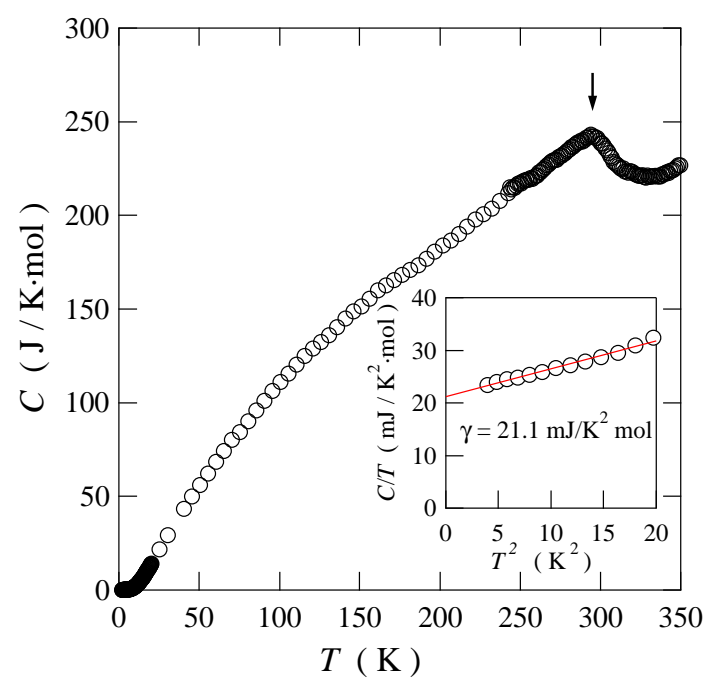

Figure 5. Temperature dependence of the heat capacity of $\mathrm{Pb}_{2} \mathrm{Re}_{2} \mathrm{O}_{6.75}$. The arrow shows the second-order phase transition temperature $T_{\mathrm{s}}$. Inset shows the plot of the heat capacity divided by temperature versus $T^{2}$.

the higher transition temperature in $\mathrm{Pb}_{2} \mathrm{Re}_{2} \mathrm{O}_{6.75}$ can be explained as a chemical pressure effect because $T_{\mathrm{s} 1}$ in $\mathrm{Cd}_{2} \mathrm{Re}_{2} \mathrm{O}_{7}$ is lowered by physical pressure [12]. The inset of Fig. 5 shows the heat capacity divided by temperature $(C / T)$ plotted against $T^{2}$. The electronic specific heat coefficient $\gamma$ is estimated as $\gamma=10.55 \mathrm{~mJ} / \mathrm{K}^{2} \mathrm{~mol} \operatorname{Re}(21.1$ $\mathrm{mJ} / \mathrm{K}^{2}$ mol f.u.). The Kadowaki-Woods ratio $A / \gamma^{2}$ in $\mathrm{Pb}_{2} \mathrm{Re}_{2} \mathrm{O}_{6.75}$ is estimated as $A / \gamma^{2}=9.9 \times 10^{-5} \mu \Omega \mathrm{cm}(\mathrm{K} \mathrm{Re} \cdot \mathrm{mol} / \mathrm{mJ})^{2}$, which is comparable to that in $\mathrm{Cd}_{2} \operatorname{Re}_{2} \mathrm{O}_{7}$ [13]. The value of $A / \gamma^{2}$ is much larger than the universal value of $1 \times 10^{-5} \mu \Omega \mathrm{cm}(\mathrm{K}$ $\mathrm{mol} / \mathrm{mJ})^{2}$, indicating that although both the resistivity and the heat capacity show the Fermi liquid-like behavior, $A$ and $\gamma$ are arisen from another contributions.

We measured XRD by changing $T$ for powdered crystal. The XRD pattern below $T_{\mathrm{s}}=294 \mathrm{~K}$ is well explained with the space group of $I 4 \bar{m} 2$, which is the same as the case of $\mathrm{Cd}_{2} \mathrm{Re}_{2} \mathrm{O}_{7}$ between $T_{\mathrm{s} 1}$ and $T_{\mathrm{s} 2}$ [14]. To investigate the order parameter of the phase transition, we carefully measured the (002) diffraction using the single crystal since this diffraction is absent in a high temperature phase whose space group is $F d \overline{3} m$. The temperature dependence of the XRD pattern around the (002) diffraction is shown in the inset of Fig. 6. The intensity of the (002) diffraction starts to grow from just below $T_{\mathrm{s}}$ with decreasing $T$, as shown in the main frame of Fig. 6 . The intensity $I$ corresponds to the square of the order parameter because $I$ is proportional to the square of the atomic displacement. The solid line in Fig. 6 shows the square of the BCS gap. The order parameter in $\mathrm{Pb}_{2} \mathrm{Re}_{2} \mathrm{O}_{6.75}$ is well described by the BCS theory over the entire temperature range as seen in many compounds exhibiting charge density wave (CDW) [15]. In $\mathrm{Pb}_{2} \mathrm{Re}_{2} \mathrm{O}_{6.75}, T_{\mathrm{s}}$ does not correspond to the CDW transition because 


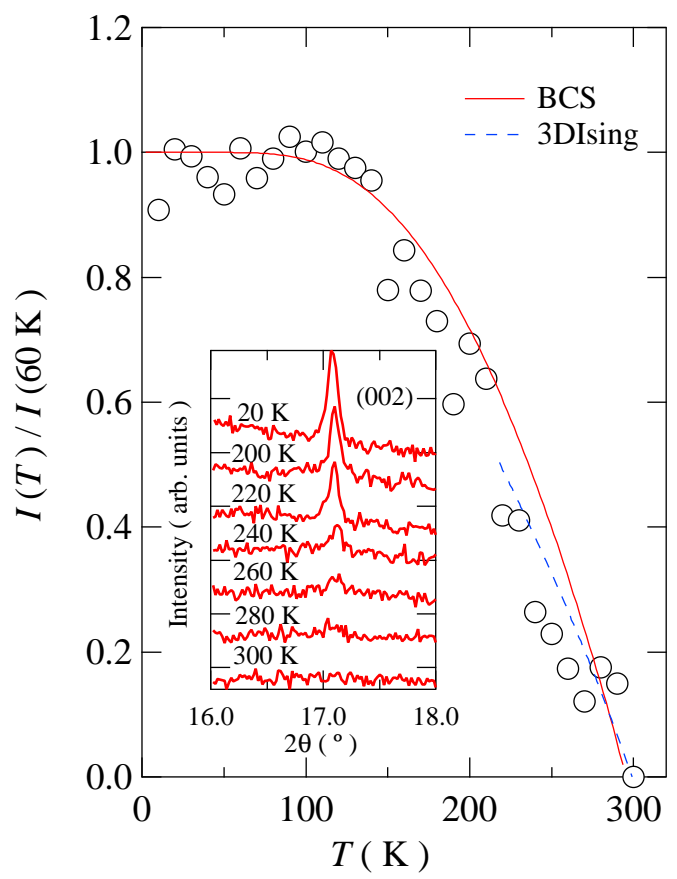

Figure 6. Temperature dependence of the normalized intensity of (002) diffraction in $\mathrm{Pb}_{2} \mathrm{Re}_{2} \mathrm{O}_{6.75}$. Solid and dashed lines show the results derived from the BCS theory and the critical behavior of the three-dimensional Ising model, respectively.

$\rho$ decreases below $T_{\mathrm{s}}$. Therefore, we propose that this second-order phase transition occurs as a result of interplay between the structural instability and the correlated electron spins to remove frustration on the pyrochlore lattice. From the results of XRD, the structure below $T_{\mathrm{s}}$ is found to be the same with that between $T_{\mathrm{s} 1}$ and $T_{\mathrm{s} 2}$ in $\mathrm{Cd}_{2} \mathrm{Re}_{2} \mathrm{O}_{7}$, where four Re atoms in a Re tetrahedra approach with each other making a quadrumer unit as shown in Fig. 1 (b). In $\mathrm{Pb}_{2} \mathrm{Re}_{2} \mathrm{O}_{6.75}$, since the magnetic ordering is not realized down to $5 \mathrm{~K}$, the rapid decrease of $\chi$ below $T_{\mathrm{s}}$ can be attributed to a spinsinglet configuration of spins in a unit of four Re atoms. This situation is quite natural because the magnetic ordering is not permitted by the strong spin frustration on the pyrochlore lattice. As already mentioned, the antiferromagnetic transition is ruled out as microscopically confirmed by the ${ }^{207} \mathrm{~Pb} \mathrm{NMR}$. The BCS-type order parameter strongly suggests the above hypothesis which is similar to a spin-Peierls transition although $\rho$ remains metallic in the whole temperature range. The ground state is the Fermi-liquid state, in which the correlation of electrons is weakened and only the Umklapp process of the conduction electrons contributes to the temperature dependence of the resistivity.

Next we discuss the critical behavior just below $T_{s}$. The dashed line in Fig. 6 shows a power law, $I \propto\left(T_{\mathrm{s}}-T\right)^{2 \beta}$ with a critical exponent $\beta=0.325$, which is the universal value in the three-dimensional Ising case. The three-dimensional Ising like behavior near $T_{\mathrm{s}}$ is similar to the case of the spin-Peierls transition in $\mathrm{CuGeO}_{3}$ [16], also suggesting 
possible realization of spin singlet quadrumers in $\mathrm{Pb}_{2} \mathrm{Re}_{2} \mathrm{O}_{6.75}$.

If we consider a projection of three-dimensional corner-sharing lattice of tetrahedron onto a two-dimensional plane, the projection maps a four spin tetrahedron onto a four spin square with additional exchange links along the diagonals, corresponding to the checkerboard antiferromagnetic model [17] as shown in Fig. 1 (b). In this model, crossed-dimer and plaquette-quadrumer valence-bond phases are predicted to occur for $J_{\times} / J \sim 0$ and $J_{\times} / J \sim 1$, respectively, where $J_{\times}$and $J$ are exchange interactions along the diagonals and the edges, respectively [17]. The former case corresponds to the Jahn-Teller distorted pyrochlore compound $\mathrm{GeCu}_{2} \mathrm{O}_{4}$ [18], and the latter to the normal pyrochlore lattice realized in the high-temperature phases of $\mathrm{Pb}_{2} \operatorname{Re}_{2} \mathrm{O}_{6.75}$ and $\mathrm{Cd}_{2} \mathrm{Re}_{2} \mathrm{O}_{7}$. Therefore, when the antiferromagnetic interactions are coupled with structural fluctuations, the quadrumerization could occur in the low-temperature phase of $\mathrm{Pb}_{2} \mathrm{Re}_{2} \mathrm{O}_{6.75}$.

\section{Summary}

A single crystal of pyrochlore $\mathrm{Pb}_{2} \mathrm{Re}_{2} \mathrm{O}_{6.75}$ was prepared by hydrothermal synthesis, and details of the physical properties were revealed for the first time. Unconventional structural second-order phase transition occurs at $T_{\mathrm{s}}=294 \mathrm{~K}$, where tetrahedra -each consisting of for four $\mathrm{ReO}_{6}$ octahedra- alternately shrink and expand as are found in $\mathrm{Cd}_{2} \mathrm{Re}_{2} \mathrm{O}_{7}$ at $T_{\mathrm{s} 1}$ [14]. The absence of magnetic ordering was confirmed microscopically by the ${ }^{207} \mathrm{~Pb}$ NMR. The order parameter at temparatures below $T_{\mathrm{s}}$ can be well described by the BCS scheme, suggesting that the phase transition originates in the interplay between correlated electrons and phonons. Such a transition possibly arises from singlet formation of the spins at the Re sites, four spins in a unit making a singlet quadrumer coupled with a structural instability similar to the spin-Peierls instability.

\section{Acknowledgments}

We thank Professor N. Yamamoto for his help in hydrothermal synthesis and useful discussions. This work was supported by Grants-in-Aid for Scientific Research from the Japan Society for Promotion of Science $(23550152,22350029)$ by Grant-in-Aid for the Global COE Program "International Center for Integrated Research and Advanced Education in Materials Science" from the Ministry of Education, Culture, Sports, Science and Technology of Japan.

\section{References}

[1] H. Sakai, K. Yoshimura, H. Ohno, H. Kato, S. Kambe, R. E. Walstedt, T. D. Matsuda, Y. Haga and Y. Onuki, J. Phys.: Condens. Matter 13, L785 (2001).

[2] M. Hanawa, Y. Muraoka, T. Tayama, T. Sakakibara, J. Yamaura and Z. Hiroi, Phys. Rev. Lett. 87, 187001 (2001).

[3] H. Sakai, M. Kato, K. Yoshimura and K. Kosuge, J. Phys. Soc. Jpn. 71, 422 (2002). 
[4] S. Lee, J. -G. Park, D. T. Adroja, D. Khomski, S. Streltsov, K. A. McEwen, H. Sakai, K. Yoshimura, V. I. Anisimov, D. Mori, R. Kanno and R. Ibberson, Nature Mater. 5, 471 (2006).

[5] A. Yamamoto, P. A. Sharma, Y. Okamoto, A. Nakao, H. Aruga Katori, S. Niitaka, D. Hashizume and H. Takagi, J. Phys. Soc. Jpn. 76, 043703 (2007).

[6] D. Mandrus, J. R. Thompson, R. Gaal, L. Forro, J. C. Bryan, B. C. Chakoumakos, L. M. Woods, B. C. Sales, R. S. Fishman and V. Keppens, Phys. Rev. B 63, 195104 (2001).

[7] T. Furubayashi, T. Matsumoto, T. Hagino and S. Nagata, J. Phys. Soc. Jpn. 63, 3333 (1994).

[8] M Isobe and Y Ueda, J. Phys. Soc. Jpn. 71, 1848 (2002).

[9] S. Fujimoto, Phys. Rev. B 67, 235102 (2003).

[10] A. M. Abakumov, R. V. Shpanchenko and E. V. Antipov, J. Solid State Chem. 138, 220 (1998).

[11] Z. Hiroi, J. Yamaura, Y. Muraoka and M. Hanawa, J. Phys. Soc. Jpn. 71, 1634 (2002).

[12] Z. Hiroi, T. Yamauchi, T. Yamada, M. Hanawa, Y. Ohishi, O. Shimomaru, M. Abliz, M. Hedo and Y. Uwatoko, J. Phys. Soc. Jpn. 71, 1553 (2002).

[13] R. Jin, J. He, S. McCall, C. S. Alexander, F. Drymiotis and D. Mandrus, Phys. Rev. B 64, 180503(R) (2001).

[14] J. Yamaura and Z. Hiroi, J. Phys. Soc. Jpn. 71, 2598 (2002).

[15] R. M. Fleming, L. F. Schneemeyer and D. E. Moncton, Phys. Rev. B 31, 899 (1985).

[16] Q. J. Harris, Q. Feng, R. J. Birgeneau, K. Hirota, G. Shirane, M. Hase and K. Uchinokura, Phys. Rev. B 52, 15420 (1995).

[17] O. A. Starykh, A. Furusaki and L. Balents, Phys. Rev. B 72, 094416 (2005).

[18] T. Yamada, Z. Hiroi, M. Takano, M. Nohara and H. Takagi, J. Phys. Soc. Jpn. 69, 1477 (1999). 